\title{
Mapping of the ${ }^{12} \mathbf{C}^{*}$ states of astrophysical interest via the ${ }^{10} \mathrm{~B}\left({ }^{3} \mathrm{He}, \mathrm{p} \alpha \alpha \alpha\right)$ Reaction
}

\author{
${\text { Martín Alcorta }{ }^{*}{ }^{\dagger} \text { H.B. Jeppesen }}^{a}$, M. J. G. Borge ${ }^{a}$, H. O. U. Fynbo ${ }^{b}$, G. Garcia ${ }^{c}$, O.

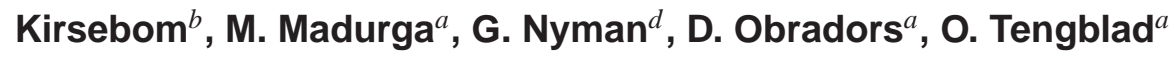 \\ ${ }^{a}$ Insto. de Estructura de la Materia, CSIC \\ E-28006 Madrid, Spain \\ E-mail: alcorta@iem.cfmac.csic.es \\ ${ }^{b}$ Department of Physics and Astronomy, University of Aarhus \\ DK-8000 Århus, Denmark \\ ${ }^{c}$ CMAM, Universidad Autónoma de Madrid \\ Cantoblanco, 28049 Madrid, Spain. \\ ${ }^{d}$ Fundamental Physics, Chalmers Univ. of Technology \\ S-41296 Göteborg, Sweden
}

\begin{abstract}
We have studied ${ }^{12} \mathrm{C}$ in full kinematics via the ${ }^{10} \mathrm{~B}\left({ }^{3} \mathrm{He}, \mathrm{p} \alpha \alpha \alpha\right)$ reaction at $2.45 \mathrm{MeV}$. In our data we have identified states in ${ }^{12} \mathrm{C}$ from the ground state up to about $18 \mathrm{MeV}$, with spins ranging from 0 to 4 . Due to the very good resolution, we are able to determine properties of these ${ }^{12} \mathrm{C}$ resonances, such as their energy, width, and spin. In this contribution preliminary results from the ongoing analysis is presented.
\end{abstract}

International Symposium on Nuclear Astrophysics - Nuclei in the Cosmos - IX

25-30 June 2006

CERN

\footnotetext{
* Speaker.

${ }^{\dagger}$ This work has been made possible by the CSIC I3P program, and is supported by the European Union under the Sixth framework project EURONS-contract number 506065.
} 


\section{Introduction}

Recently our collaboration has studied ${ }^{12} \mathrm{C}^{*}$ states fed by the $\beta$ decay of ${ }^{12} \mathrm{~N}$ and ${ }^{12} \mathrm{~B}[1]$. The ${ }^{3} \mathrm{He}+{ }^{10} \mathrm{~B}$ reaction study presented here allows us to gain complementary information on the ${ }^{12} \mathrm{C}$ resonances compared to what is known from $\beta$ decay experiments because we populate states not accessible to $\beta$ decay due to energetic limitations and selection rules. For example, $\beta$ decay studies will only populate the positive parity states, while the ${ }^{3} \mathrm{He}+{ }^{10} \mathrm{~B} \rightarrow \mathrm{p}+{ }^{12} \mathrm{C}^{*}$ channel can give us access to more states in ${ }^{12} \mathrm{C}^{*}$ that may be of relevance in the triple-alpha process responsible for helium burning in stars.

Although the Hoyle resonance dominates the triple reaction rate at the most relevant astrophysical temperatures of $0.1<T_{9}<2.0$, at higher temperatures other natural parity states such as $0^{+}, 1^{-}, 2^{+}$, and $3^{-}$may play a more dominant role [2]. We have identified both the $3^{-} 9.64 \mathrm{MeV}$ state and the $1^{-} 10.8 \mathrm{MeV}$ state, and can extract information on their break-up. By studying the break-up of the three alphas from ${ }^{12} \mathrm{C}$, we can gain information on resonances near the triple alpha threshold which may help clarify the rate of ${ }^{12} \mathrm{C}$ production at high temperatures.

The ${ }^{10} \mathrm{~B}\left({ }^{3} \mathrm{He}, \mathrm{p} \alpha \alpha \alpha\right)$ reaction was studied once before in the 60 's, but with limited technology. The $\mathrm{p}+{ }^{12} \mathrm{C}^{*}$ channel was identified on-line from the image on an oscilloscope with two particles preselected in hardware coincidence at given angles [3]. With the development of segmented detectors and faster electronics, we have detected 4-particle coincidences in our setup at the $5 \mathrm{MV}$ tandetron in Madrid with a much improved angular resolution.

\section{Experiment}

The Experiment was performed at the Centro de Microanálisis de Materiales (CMAM), located in the campus of the Universidad Autónoma de Madrid (Spain). It houses the first Coaxial High Current Tandetron Accelerator of 5MV using the Cockroft-Walton power supply system [4]. This method allows for very stable beams, making the CMAM accelerator an ideal place for these type of experiments.

The experimental set-up consisted of one $60 \mu \mathrm{m}$ and one $69 \mu \mathrm{m}$ DSSSD [5, 6], the latter backed by two Si-detectors of $1473 \mu \mathrm{m}$ and $1000 \mu \mathrm{m}$, and the former with just one Si-detector of $1498 \mu \mathrm{m}$. The DSSSDs have 16 x 16 perpendicular strips, giving a granularity of $2563 \times 3 \mathrm{~mm}$ pixels, and covering a solid angle of 1/9th of $4 \pi$. The 2-particle detection efficiency was about $1 \%$, and the 4-particle detection efficiency was approximately $0.015 \%$. The reaction took place with a beam of ${ }^{3} \mathrm{He}$ at $2.45 \mathrm{MeV}$ on a target of ${ }^{10} \mathrm{~B}$ with a thickness of $20 \mathrm{mg} / \mathrm{cm}^{2}$.

The combination of a thin DSSSD backed with a thick Si-detector were used for particle identification. Protons produced in the reaction can reach energies of up to $21 \mathrm{MeV}$, thereby depositing only a small part of their energy in the DSSSD. The alpha particles, however, will be stopped almost entirely in the DSSSD, due to their greater mass and lower energy. By selecting the protons produced in the reaction, we can reproduce the level scheme of ${ }^{12} \mathrm{C}$. Figure 1 shows the spectrum of excited states in ${ }^{12} \mathrm{C}$ together with the experimental data. This figure demonstrates both the advantages and disadvantages of studying this nucleus via a reaction: it populates all states in ${ }^{12} \mathrm{C}$, yet as a result, the spectrum is not as clean as in $\beta$ decay studies. 


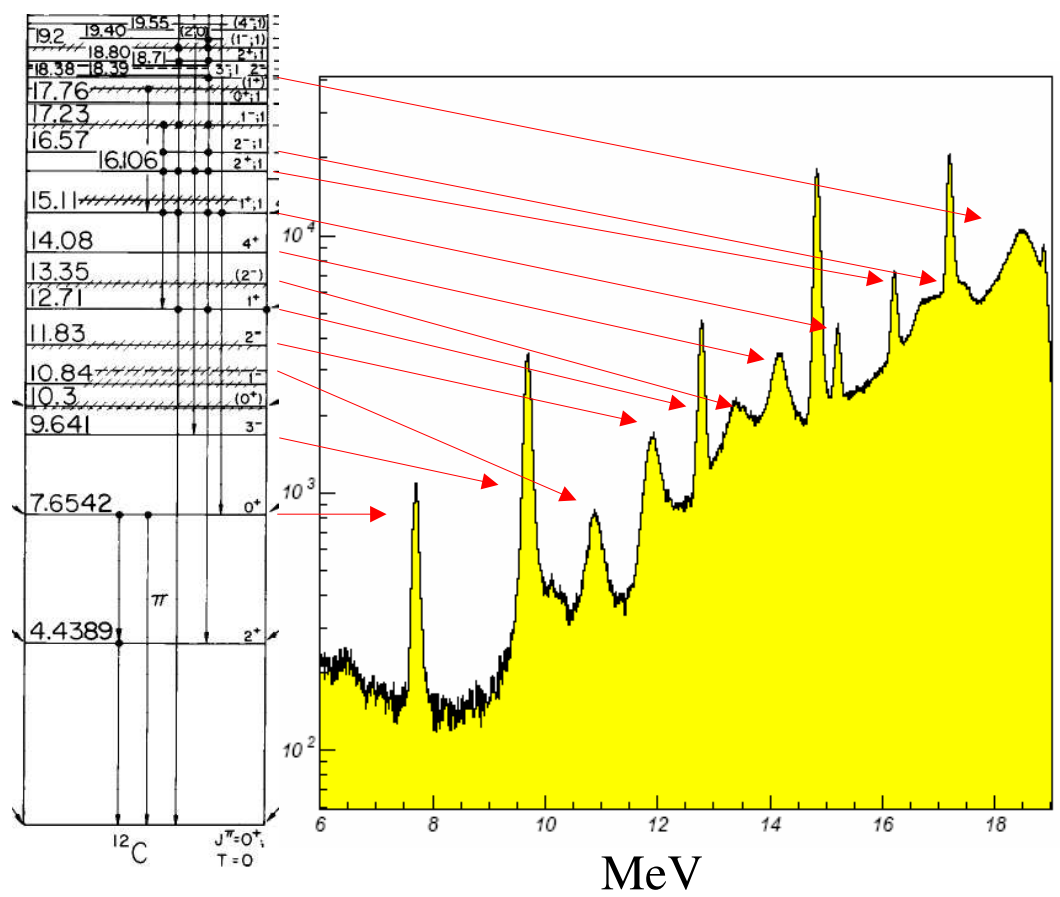

Figure 1: The level scheme of the excited states in ${ }^{12} \mathrm{C}$ on the left [7] is shown together with the spectrum obtained in this experiment (right). The protons were identified in each telescope. The different proton peaks are related to states in ${ }^{12} \mathrm{C}$, which are indicated by the arrows.

\section{Results}

By selecting on the protons produced in the ${ }^{3} \mathrm{He}+{ }^{10} \mathrm{~B} \rightarrow \mathrm{p}+{ }^{12} \mathrm{C} *$ reaction, we can calculate the excitation spectrum of ${ }^{12} \mathrm{C}$, as shown in Figure 1 together with the literature values of the resonance states. With these results, we can easily select the different resonances populated in the reaction for further analysis. The use of position sensitive detectors gives information on the momentum of the alpha particles produced from the break-up of ${ }^{12} \mathrm{C}$, allowing us to reconstruct the momentum of a third alpha particle in cases where only two alpha particles were detected. Once we have the momentum of each alpha particle, and assuming sequential decay through ${ }^{8} \mathrm{Be}$, we can determine whether the break-up of each state went via the $0^{+}$ground state in ${ }^{8} \mathrm{Be}$ and/or the $2^{+}$in ${ }^{8} \mathrm{Be}$. This is done using the following relation:

$$
\begin{aligned}
& E\left({ }^{8} \mathrm{Be}\right)=\frac{\left|P_{1}\right|^{2}+\left|P_{2}\right|^{2}-2 \cdot\left|P_{1}\right| \cdot|P 2| \cdot \cos \theta}{4 m_{\alpha}} \\
& \cos \theta=\frac{{\overrightarrow{P_{x 1}}}^{2} \cdot{\overrightarrow{P_{x 2}}}^{2}+{\overrightarrow{P_{y 1}}}^{2} \cdot{\overrightarrow{P_{y 2}}}^{2}+{\overrightarrow{P_{z 1}}}^{2} \cdot{\overrightarrow{P_{z 2}}}^{2}}{\left|P_{1}\right| \cdot\left|P_{2}\right|}
\end{aligned}
$$

The ${ }^{8} \mathrm{Be}$ energy is calculated by using the three possible pairs of alpha particles which could make up an intermediate ${ }^{8} \mathrm{Be}$ nucleus. Once the energy in ${ }^{8} \mathrm{Be}$ is calculated, one can select those alpha pairs and identify which state was populated in ${ }^{8} \mathrm{Be}$. The ground state peak is at $0.09 \mathrm{MeV}$, while the broad $2^{+}(\Gamma=1.37 \mathrm{MeV})$ is at $3.06 \mathrm{MeV}$. For highly excited states in ${ }^{12} \mathrm{C}$, the $4^{+}$state in 


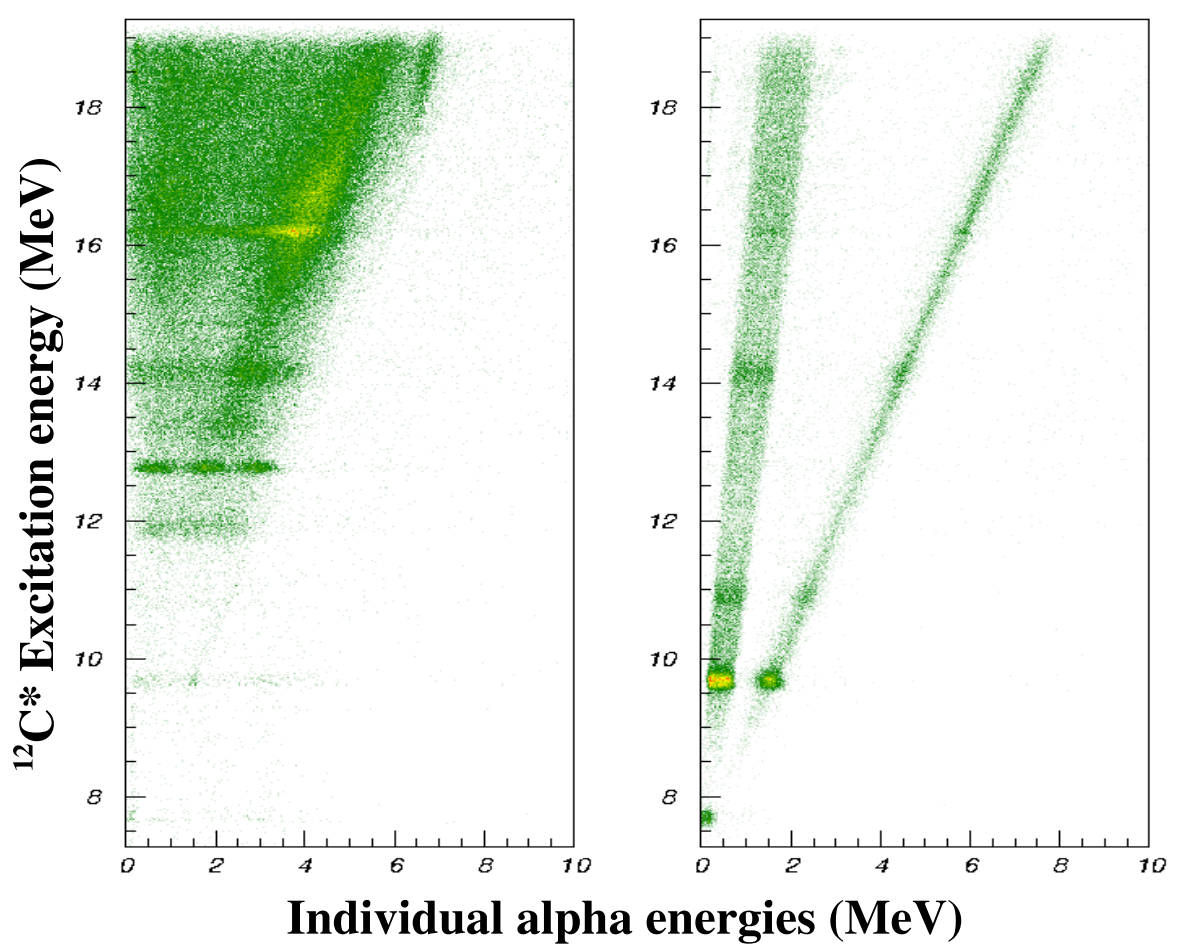

Figure 2: Here we see two plots of individual alpha energies in the $\mathrm{x}$-axis plotted vs. the ${ }^{12} \mathrm{C}$ excitation energy on the y-axis. On the left we see the states that break-up via the ${ }^{8} \mathrm{Be} 2^{+}$state, and on the right we can see the break-up via the ${ }^{8} \mathrm{Be} 0^{+}$ground state.

${ }^{8} \mathrm{Be}$ should also be considered. Figure 2 shows plots of the three alpha particles decaying through these two different states in ${ }^{8} \mathrm{Be}$. These plots represent the ${ }^{12} \mathrm{C}$ excitation energy versus the individual alpha particle energy (in the ${ }^{12} \mathrm{C}$ center of mass frame).

Using these methods, we separate break-ups via the ${ }^{8} \mathrm{Be} 2^{+}$and ground state, and then plot the individual alpha energies for each particular state in ${ }^{12} \mathrm{C}$. By comparing these plots to simulations, we can attribute spins and parities to the states we have identified. The break-up through the ${ }^{8} \mathrm{Be} 0^{+}$ state gives information on which alpha particle is emitted first. Angular correlations between the proton and the first alpha particle can then be used to determine the spin of the state. The picture gets more complicated when the break-up involves the ${ }^{8} \mathrm{Be} 2^{+}$state since interference among the three alpha particles will have to be considered [8].

\section{Outlook}

It is important to perform more analysis on the individual states in ${ }^{12} \mathrm{C}$ and better determine their break-up mechanisms. Using the methods mentioned earlier, we can determine if some states, such as the $9.65 \mathrm{MeV}$ state, break-up via the narrow $0^{+}{ }^{8} \mathrm{Be}$ resonance or via its tail. These results could modify the reaction rate of the triple-alpha reaction, depending on the break-up route. In a recent paper, the possibility of the $3^{-} 9.65 \mathrm{MeV}$ state and the $1^{-} 10.8 \mathrm{MeV}$ state having an influence on the reaction rate are considered [2]. 
Some preliminary studies of the ${ }^{9} \mathrm{~B}$ nucleus from the ${ }^{9} \mathrm{~B}^{*}+\alpha$ channel have been studied as well, including identification of the ground state and the $2.34 \mathrm{MeV}$ excited state. Studying ${ }^{9} \mathrm{~B}^{*}$ can give us information on the states in ${ }^{9} \mathrm{Be}^{*}$, its mirror nucleus, which influences the $\alpha \alpha \mathrm{n}$ reaction and therefore also contributes to helium burning.

This experiment was repeated in the spring of 2006 with an improved detectors setup as well as more beam time. Instead of using two telescope detectors, the target was surrounded by 4 telescope detectors, covering both forward and backward angles. This will greatly increase our multi-particle detection efficiency, thereby greatly increasing our statistics.

\section{References}

[1] H.O.U. Fynbo et al., Nature 433, (2005) 137.

[2] L.R. Buchmann and C.A. Barnes, Nuclear reactions in stellar helium burning and later hydrostatic burning stages, Nucl. Phys. A, in press, corrected proof (2005).

[3] M.A. Waggoner et al., A study of the reaction ${ }^{10} B\left({ }^{3} H e, p \alpha \alpha \alpha\right)$, Nucl. Phys. 88 (1966) 81.

[4] O. Enguita et al., Centro de Micro-Análisis de Materiales (Madrid, Spain), SNEAP 2005.

[5] U.C. Bergmann et al., Use of Si strip detectors for low-energy particles in compact geometry, Nucl. Inst. and Methods A 515 (2003) 657.

[6] O. Tengblad et al., Novel thin window design for a large-area silicon strip detector, Nucl. Inst. and Methods A 525 (2004) 458.

[7] F. Ajzenberg-Selove, Energy levels of light nuclei A=11-12, Nucl. Phys. A 506 (1990) 1.

[8] H.O.U. Fynbo et al., Clarification of the three-body decay of ${ }^{12} \mathrm{C}(12.71 \mathrm{MeV})$, Phys. Rev. Lett. 91 (2003) 82502. 